\title{
Very long chain acyl-CoA dehydrogenase deficiency
}

INSERM

\section{Source}

INSERM. (1999). Orphanet: an online rare disease and orphan drug data base. Very long chain acyl-COA dehydrogenase deficiency. ORPHA:26793

Very long-chain acyl-CoA dehydrogenase (VLCAD) deficiency (VLCADD) is an inherited disorder of mitochondrial long-chain fatty acid oxidation with a variable presentation including: cardiomyopathy, hypoketotic hypoglycemia, liver disease, exercise intolerance and rhabdomyolysis. 